\title{
Quasi-Invariant Measures on the Orthogonal Group over the Hilbert Space
}

By

\author{
Hiroaki SHIMOMURA*
}

\section{§1. Introduction}

Let $H$ be a real separable Hilbert space and $O(H)$ be the orthogonal group over $H$. In this paper, we shall discuss left, right or both translationally quasi-invariant probability measures on a $\sigma$ field $\mathfrak{B}$ derived from the strong topology on $O(H)$. Invariant (rather than quasi-invariant) measures have been considered by several authors. For example in [3], [7] and [4] such measures were constructed as suitable limits of Haar measures on $O(n)$ by methods of Schmidt's orthogonalization or of Cayley transformation. And in [6] some approach based on Gaussian measures on infinite-dimensional linear spaces was attempted. However these measures are defined on larger spaces rather than $O(H)$ and invariant under a sense that " $O(H)$ acts on these spaces." This is reasonable, because it is impossible to construct measures on $O(H)$ which are invariant under all translations of elements of $G$, if $G$ is a suitably large subgroup of $O(H)$. For example, let $e_{1}, \cdots, e_{n}, \cdots$ be a c.o.n.s. in $H$, and for each $n$ consider a subgroup consisting of $T \in O(H)$ which leaves $e_{p}$ invariant for all $p>n$. We may identify this subgroup with $O(n)$. Put $O_{0}(H)=\cup_{n=1}^{\infty} O(n)$. Then $O_{0}(H)$-invariant finite measure does not exist on $O(H)$. (See, [6]). However replacing invariance with quasi-invariance, the above situation becomes somewhat different. One but main purpose of this paper is to indicate this point. We will show that "there does not exist any $\sigma$-finite G-quasi-invariant measure on $\mathfrak{B}$, as far as $G$ acts transitively on the unit sphere $S$ of $H$. While $O_{0}(H)$-quasi-invariant probability measures certainly exist.

Communicated by S. Matsuura, December 9, 1982.

* Department of Mathematics, Fukui University, Fukui 910, Japan. 
We can construct one of them by the Schmidt's orthogonalization method using a suitable family of measures on $H$." In the remainder parts, we will state basic properties, especially ergodic decomposition of $O_{0}(H)$-quasi-invariant probability measures. These arguments are carried out in parallel with them for quasi-invariant measures on linear spaces. (See, [5]).

\section{§ 2. Non Existence of $\boldsymbol{G}$-Quasi-Invariant Measures}

Let $e_{1}, \cdots, e_{n}, \cdots$ be an arbitrarily fixed c.o.n.s. in $H$, and define a metric $d(\cdot, \cdot)$ on $O(H)$ such that $d(U, V)=\sum_{n=1}^{\infty} 2^{-n}\left\{\left\|U e_{n}-V e_{n}\right\|+\right.$ $\left.\left\|U^{-1} e_{n}-V^{-1} e_{n}\right\|\right\}$, where $\|\cdot\|$ is the Hilbertian norm on $H$. A map $U \in O(H) \longmapsto\left(\left(U e_{1}, \cdots, U e_{n}, \cdots\right),\left(U^{-1} e_{1}, \cdots, U^{-1} e_{n}, \cdot \cdot\right)\right) \in H^{\infty} \times H^{\infty}$ is a into homeomorphism from $(O(H), d)$ to $H^{\infty} \times H^{\infty}$ equipped with the product-topology. Hence $(O(H), d)$ is a separable metric space. The topology derived from $d$ coincides with the strong topology on $O(H)$, so $(O(H), d)$ is a topological group and $\mathfrak{B}$ is a $\sigma$-field generated by open sets of $(O(H), d)$. Moreover, since inverse terms $\| U^{-1} e_{n}$ $V^{-1} e_{n} \|$ are added to the definition of $d,(O(H), d)$ is a complete metric space and therefore a Polish space. Now let $\mu$ be a measure on $\mathfrak{B}$ and $T \in O(H)$. We shall define measures $L_{T} \mu\left(R_{T} \mu\right)$ by $L_{T} \mu(B)=\mu\left(T^{-1} \cdot B\right) \quad\left(R_{T} \mu(B)=\mu\left(B \cdot T^{-1}\right)\right)$ for all $B \in \mathfrak{B}$, and call them left translation (right translation) of $\mu$ by $T$, respectively. If for a fixed subgroup $G \subset O(H), L_{T} \mu\left(R_{T} \mu\right)$ is equivalent to $\mu, L_{T} \mu \simeq \mu$, for all $T \in G, \mu$ is said to be left (right) $G$-quasi-invariant, respectively. Left and right $G$-quasi-invariant measures are defined in a similar manner. Since results for right $G$-quasi-invariant measures are formally derived from them for left $G$-quasi-invariant measures, we shall omit the "right" case for almost everywhere.

Theorem 1. There does not exist any left (right) G-quasi-invariant $\sigma$-finite measure on $\mathfrak{B}$, as far as $G$ acts transitively on the unit sphere $S$ of $H$.

Proof. Suppose that it would be false, and let $\mu$ be a such one of left $G$-quasi-invariant measures. As $(O(H), d)$ is a Polish space, there exists a sequence of compact sets $\left\{K_{n}\right\}$ of $(O(H), d)$ such 
that $\mu\left(K_{n}\right)>0(n=1,2, \cdots)$ and $\mu\left(\cap_{n=1}^{\infty} K_{n}^{c}\right)=0$. From the assumption, we have $0<\mu\left(g K_{1}\right)=\mu\left(\cup_{n=1}^{\infty} K_{n} \cap g K_{1}\right)$ for all $g \in G$, and therefore $K_{n} \cap g K_{1} \neq \phi$ for some $n$. It follows that $G \subset \cup_{n=1}^{\infty} K_{n} K_{1}^{-1}$. Take an $e \in S$ and consider a continuous map $f ; U \in(O(H), d) \longmapsto U e \in S$. Then we have $S=f(G)=\cup_{n=1}^{\infty} f\left(K_{n} K_{1}^{-1}\right) \subset S$. Hence $S$ is a $\sigma$-compact set. However it is impossible in virtue of Baire's category theorem.

Q.E.D.

\section{§3. A Construction of $O_{0}(H)$-Quasi-Invariant Measures}

As it was stated in the Introduction, let us form $O_{0}(H)$ from an arbitrarily fixed c.o.n.s. $e_{1}, \cdots, e_{n}, \cdots$. For the purpose of the above title, it is enough to regard $H$ as $\ell^{2}$ and the above base as $e_{n}=(0, \cdots$, $\stackrel{n}{1}, 0 \cdots) \in \ell^{2}$. First we shall consider left quasi-invariant probability measures, and shall state some lines fo the construction. Let $\tilde{x}=\left(x_{1}\right.$, $\left.\cdots, x_{n}, \cdots\right)$ be a sequence of $\ell^{2}$. If they are linearly independent, we have an orthonormal system $G\left(x_{1}\right), G\left(x_{1}, x_{2}\right), \cdots, G\left(x_{1}, \cdots, x_{n}\right), \cdots$, operating on $x_{1}, \cdots, x_{n}, \cdots$ Schmidt's orthogonalization process. Moreover, they form a c.o.n.s., if a subspace $L(\tilde{x})$ spanned by $x_{1}$, •, $x_{n}, \cdots$ is dense in $\ell^{2}$. And then we can define an orthogonal operator $U(\tilde{x})$ on $\ell^{2}$ as $e_{n} \longmapsto G\left(x_{1}, \cdots, x_{n}\right)$ for all $n$. Now for each $T \in O\left(\ell^{2}\right)$, we shall define a map $\widetilde{T}$ on the $\ell^{2}$-sequence space $\left(\ell^{2}\right)^{\infty}$ such that $\widetilde{T}\left(x_{1}, \cdots, x_{n}, \cdots\right)=\left(T x_{1}, \cdots, T x_{n}, \cdots\right)$. Then it is easy to see that $L_{T} \circ U=U \circ \tilde{T}$, namely, $T U(\tilde{x})=U(\tilde{T} \tilde{x})$. Hence one of left $O_{0}(H)-$ quasi-invariant measures $\lambda$ on $\mathfrak{B}$ is defined as $\lambda(B)=\tilde{\nu}(\tilde{x} \mid U(\tilde{x}) \in B)$ for all $B \in \mathfrak{B}$, if we can construct a probability measure $\tilde{\mathcal{L}}$ on the usual Borel field $\mathfrak{B}\left(\left(\ell^{2}\right)^{\infty}\right)$ on $\left(\ell^{2}\right)^{\infty}$ satisfying following three properties,

(a) $x_{1}, x_{2}, \cdots, x_{n}, \cdots$ are linearly independent for $\tilde{\mathbf{v}}-a . e . \tilde{x}=\left(x_{1}\right.$, $\left.\cdots, x_{n}, \cdots\right)$,

(b) “ $L(\tilde{x})$ is dense in $\ell^{2}$ " holds for $\tilde{\nu}-a \cdot e . \tilde{x}$,

(c) $\tilde{T} \tilde{\nu}\left(\widetilde{T} \tilde{\nu}(B)=\tilde{\nu}\left((\widetilde{T})^{-1}(B)\right)\right.$ for all $\left.B \in \mathfrak{B}\left(\left(\ell^{2}\right)^{\infty}\right)\right)$ is equivalent to $\tilde{\mathcal{v}}$ for all $T \in O_{0}\left(\ell^{2}\right)$.

Now let $p$ be a probability measure on $\mathfrak{B}\left(\mathbb{R}^{1}\right)$ which is equivalent to the Lebesgue measure and satisfies $\int_{-\infty}^{\infty} t^{-2} d p(t)=1$. 1 -dimensional Gaussian measures with mean 0 and variance $c$ will be denoted by $g_{c}$. And take positive sequences $\left\{v_{n}\right\}_{n=2}^{\infty}$ and $\left\{c_{n}\right\}_{n=2}^{\infty}$ such that 


$$
\sum_{n=1}^{\infty} n v_{n+1}+\sum_{n=1}^{\infty} \sum_{j=n \perp 1}^{\infty} c_{j}^{2}<1 .
$$

Then for each $n$, a measure of product-type $\mu_{n}=\underbrace{g_{v_{n}} \times \cdots \times g_{v_{n}}}_{v_{n}-1 \text { times }} \times p \times$ $g_{c_{n+1}^{2}}^{2} \times \cdots \times g_{c_{j}^{2}} \times \cdots$ is defined on $\mathfrak{B}\left(\ell^{2}\right)$, in virtue of the choice of $\left\{c_{n}\right\}$. Moreover, from the rotational-invariance of $g_{v_{n}} \times \cdots \times g_{v_{n}}, \mu_{n}$ is $O(n-1)$-invariant for all $n$. Now let us consider a measure of product-type $\tilde{\mu}=\mu_{1} \times \cdots \times \mu_{n} \times \cdots$ on $\mathfrak{B}\left(\left(\ell^{2}\right)^{\infty}\right)$. It is fairly easy that $\tilde{\mu}$ satisfies (a). Since for all $n$ and for all $T \in O(n-1)$, we have

$$
\begin{aligned}
\widetilde{T} \tilde{\mu} & =T \mu_{1} \times \cdots \times T \mu_{n-1} \times T \mu_{n} \times \cdots \times T \mu_{j} \times \cdots \\
& =T \mu_{1} \times \cdots \times T \mu_{n-1} \times \mu_{n} \times \cdots \times \mu_{j} \times \cdots \\
& \simeq \mu_{1} \times \cdots \times \mu_{n} \times \cdots=\tilde{\mu},
\end{aligned}
$$

so $\tilde{\mu}$ satisfies (c) too. We shall consider for (b). Let $\langle\cdot$, $\cdot\rangle$ be the scalar product on $\ell^{2}$. Then,

$$
\begin{aligned}
\int_{y \in e^{2}} & \left.\|<y, e_{n}\right\rangle^{-1} y-e_{n}||^{2} d \mu_{n}(y) \\
= & \left.\int\left\langle y, e_{n}\right\rangle^{-2} \sum_{j \neq n}<y, e_{j}\right\rangle^{2} d \mu_{n}(y) \\
= & (n-1) v_{n}+\sum_{j=n+1}^{\infty} c_{j}^{2},
\end{aligned}
$$

it follows that

$$
\begin{aligned}
& \int_{\left(\ell^{2}\right)^{\infty}} \sum_{n=1}^{\infty}\left\|<x_{n}, e_{n}>^{-1} x_{n}-e_{n}\right\|^{2} d \tilde{\mu}(\tilde{x}) \\
& \quad=\sum_{n=1}^{\infty} n v_{n+1}+\sum_{n=1}^{\infty} \sum_{j=n+1}^{\infty} c_{j}^{2}<1 .
\end{aligned}
$$

Hence putting

$$
E=\left\{\tilde{x}\left|\sum_{n=1}^{\infty}\right| \mid\left\langle x_{n}, e_{n}>^{-1} x_{n}-\left.e_{n}\right|^{2}<1\right\},\right.
$$

we have $\tilde{\mu}(E)>0$. Thus for all $\tilde{x} \in E, L(\tilde{x})$ is dense in $\ell^{2}$ by the following lemma.

Lemma 1. Suppose that $\sum_{n=1}^{\infty}|| t_{n}-\left.e_{n}\right|^{2}<1$ for a sequence $\left\{t_{n}\right\} \subset \ell^{2}$. Then a subspace spanned by $t_{1}, \cdots, t_{n}, \cdots$ is dense in $\ell^{2}$.

Proof. By the assumption, we can define an operator $A$ such that $A e_{n}=t_{n}$ for all $n$ and $I-A$ is a Hilbert-Schmidt operator whose Hilbert-Schmidt norm is strictly less than 1 . Hence we have $\|I-A\|_{o p}$ $<1$. It implies $A$ is an isomorphic operator. Consequently, $A e_{1}, \cdots$, $A e_{n}, \cdots$ span a dense linear subspace.

Q. E. D.

At the same time we shall prove the measurability of the set 
$\{\tilde{x} \mid L(\tilde{x})$ is dense $\} \equiv F$. Consider a set $\left(\ell^{2}\right)^{\infty} \times S \supset \Omega \equiv\left\{(\tilde{x}, a) \mid<x_{n}\right.$, $a>=0$ for all $n\}$ and let $p$ be a projection to the first coordinate. It is evident that $F^{c}=p(\Omega)$, and the later is a Souslin set. Therefore $F$ is universally-measurable. As $\tilde{\mu}(F) \geqq \tilde{\mu}(E)>0$, so we can put $\tilde{\nu}(B)$ $=\frac{\tilde{\mu}(B \cap F)}{\tilde{\mu}(F)}$ for all $B \in \mathfrak{B}\left(\left(\ell^{2}\right)^{\infty}\right)$. Clearly, $\tilde{\nu}$ satisfies (a) and (b). Moreover (c) is also satisfied, because $F$ is an invariant set for all $\widetilde{T}, T \in O\left(\ell^{2}\right)$. By the above, there exist left $O_{0}(H)$-quasi-invariant probability measures on $\mathfrak{B}$. Next, if we wish to construct left and right $O_{0}(H)$-quasi-invariant measures, we shall prepare such $\tilde{\nu}_{1}$ and $\tilde{\nu}_{2}$ and form a product-measure $\tilde{\nu}_{1} \times \tilde{\nu}_{2}$ on $\left(\ell^{2}\right)^{\infty} \times\left(\ell^{2}\right)^{\infty}$. Then for $\tilde{\nu}_{1} \times \tilde{\nu}_{2}$-a. e. $(\tilde{x}, \tilde{y}), U(\tilde{x}, \tilde{y}) ; G\left(x_{1}, \cdots, x_{n}\right) \longmapsto G\left(y_{1}, \cdots, y_{n}\right)(n=1,2 \cdots)$ is an orthogonal operator on $\ell^{2}$ which satisfies $U(\widetilde{T} \tilde{x}, \tilde{S} \tilde{y})=S U(\tilde{x}, \tilde{y}) T^{-1}$ for all $T, S \in O\left(\ell^{2}\right)$. It follows by similar arguments that a measure $\lambda=U\left(\tilde{\nu}_{1} \times \tilde{\nu}_{2}\right)$ on $\mathfrak{B}$ is a left and right $O_{0}\left(\ell^{2}\right)$-quasi-invariant probability measure.

\section{§4. Basic Results and Ergodic Decomposition of $\boldsymbol{O}_{0}(\boldsymbol{H})$-Quasi-Invariant Measures}

From now on, we put $\mathfrak{A}_{n}=\{E \in \mathfrak{B} \mid T \cdot E=E$ for all $T \in O(n)\}$,

$$
\begin{aligned}
& \mathfrak{B}_{n}=\{E \in \mathfrak{B} \mid T \cdot E \cdot S=E \text { for all } T, S \in O(n)\} \quad(n=1,2, \cdots), \\
& \mathfrak{A}_{\infty}=\left\{E \in \mathfrak{B} \mid T \cdot E=E \text { for all } T \subseteq O_{0}(H)\right\}
\end{aligned}
$$

and

$$
\mathfrak{B}_{\infty}=\left\{E \in \mathfrak{B} \mid T \cdot E \cdot S=E \text { for all } T, S \in O_{0}(H)\right\} .
$$

Then we have $\mathfrak{A}_{1} \supset \cdots \supset \mathfrak{A}_{n} \supset \cdots, \mathfrak{B}_{1} \supset \cdots \supset \mathfrak{B}_{n} \supset \cdots, \cap_{n=1}^{\infty} \mathfrak{A}_{n}=\mathfrak{A}_{\infty}$, and $\cap_{n=1}^{\infty} \mathfrak{B}_{n}=\mathfrak{B}_{\infty} . \quad \mathfrak{A}_{\infty}\left(\mathfrak{B}_{\infty}\right)$ plays an essential role for left (left and right) $\mathrm{O}_{0}(H)$-quasi-invariant measures.

Lemma 2. (a) Let $\mu$ be a left $O_{0}(H)$-quasi-invariant probability measure on $\mathfrak{B}$, and let $E \in \mathfrak{B}$ satisfy $\mu(E \ominus T \cdot E)=0$ for all $T \in O_{0}(H)$. Then there exists an $E_{0} \in \mathfrak{A}_{\infty}$ such that $\mu\left(E \ominus E_{0}\right)=0$.

(b) Let $\mu$ be a left and right $O_{0}(H)$-quasi-invariant probability measure on $\mathfrak{B}$, and let $E \in \mathfrak{B}$ satisfy $\mu(E \ominus T \cdot E \cdot S)=0$ for all $T, S \in$ $O_{0}(H)$. Then there exists an $E_{0} \in \mathfrak{B}_{\infty}$ such that $\mu\left(E \ominus E_{0}\right)=0$.

Proof. (a) Put $f_{n}(U)=\int_{O(n)} \chi_{E}(T \cdot U) d T$, where $d T$ is the normal- 
ized Haar measure on $O(n)$ and $\chi_{E}$ is the indicator function of $E$. Then $f_{n}(U)$ is an $O(n)$-invariant function and

$$
\begin{aligned}
& \int\left|f_{n}(U)-\chi_{E}(U)\right| d \mu(U) \\
& \quad \leqq \iint\left|\chi_{E}(T \cdot U)-\chi_{E}(U)\right| d T d \mu(U) \\
& \quad=\int_{O(n)} \mu\left(E \ominus T^{-1} \cdot E\right) d T=0 .
\end{aligned}
$$

Hence we have $f_{n}(U)=\chi_{E}(U)$ for $\mu$-a. e. $U$. Put $f(U)=\lim _{n} f_{n}(U)$, if the limit exists and $f(U)=0$, otherwise. Since $f(U)$ is $O_{0}(H)$-invariant, so putting $E_{0}=\{U \mid f(U)=1\}$, it holds $\mu\left(E \ominus E_{0}\right)=0$.

(b) It is carried out in a similar manner, only changing the integral into $\iint_{O(n) \times O(n)} \chi_{E}(T \cdot U \cdot S) d T d S . \quad$ Q. E. D.

Lemma 3. Let $\mu$ be a left $O_{0}(H)$-quasi-invariant probability measure on $\mathfrak{B}$. Then for any $B \in \mathfrak{B}$ there exists a countable set $\left\{T_{n}\right\}_{n=1}^{\infty} \subset$ $O_{0}(H)$ such that $\hat{B} \equiv \cup_{n=1} T_{n} \cdot B$ satisfies $\mu(T \cdot \hat{B} \ominus \hat{B})=0$ for all $T \in$ $O_{0}(H)$. If $\mu$ is a left and right $O_{0}(H)$-quasi-invariant probability measure, then it holds $\mu(T \cdot \hat{B} \cdot S \ominus \hat{B})=0$ for all $T, S \in O_{0}(H)$, replacing the above set with $\hat{B}=\cup_{n=1} T_{n} \cdot B \cdot S_{n}$ for some $\left\{T_{n}\right\}_{n},\left\{S_{n}\right\}_{n} \subset O_{0}(H)$.

Proof. As $L_{\mu}^{1}(O(H))$ is separable, we can take a countable dense set $\left\{\chi_{T_{n} \cdot B}(U)\right\}_{n=1}^{\infty}$ of $\left\{\chi_{T \cdot B}(U)\right\}_{T \in O_{0}(H)}$ in the left case and $\left\{\chi_{T_{n} \cdot B \cdot S_{n}}(U)\right\}_{n=1}^{\infty}$ of $\left\{\chi_{T \cdot B \cdot S}(U)\right\}_{T, S \in O_{0}(H)}$ in the left and right case. It is easily checked that $U_{n=1} T_{n} \cdot B$ and $U_{n=1} T_{n} \cdot B \cdot S_{n}$ are desired ones respectively.

Q. E. D.

Proposition 1. Two left $O_{0}(H)$-quasi-invariant probability measures $\mu$ and $\nu$ are equivalent, if and only if $\mu \simeq \nu$ on $\mathfrak{H}_{\infty}$. In the case of left and right $O_{0}(H)$-quasi-invariant measures, it is necessary and sufficient that they are equivalent on $\mathfrak{B}_{\infty}$.

Proof. The necessity is obvious. So let $\mu$ and $\nu$ be left $O_{0}(H)-$ quasi-invariant and suppose that they are not equivalent, for example, $\mu(B)>0$ and $\nu(B)=0$ for some $B \in \mathfrak{B}$. Then applying Lemma 3 for $\mu$, there exists $\left\{T_{n}\right\}_{n} \subset O_{0}(H)$ such that $\hat{B}=\cup_{n=1} T_{n} \cdot B$ satisfies $\mu(T \cdot \hat{B}$ $\ominus \hat{B})=0$ for all $T \in O_{0}(H)$. Clearly we have $\nu(T \cdot \hat{B} \ominus \hat{B})=0$ for all 
$T \in O_{0}(H)$. Thus applying Lemma 2 for $\lambda=2^{-1}(\mu+\nu)$, there exists a n $A \in \mathfrak{A}_{\infty}$ such that $\lambda(A \ominus \hat{B})=0$. It follows that $\mu(A)=\mu(\hat{B})>0$ and $\nu(A)=\nu(\hat{B})=0$. Therefore $\mu$ and $\nu$ are not equivalent on $\mathfrak{A}_{\infty}$. The left and right case is discussed in a similar way.

Q.E.D.

Now we shall introduce a notion of ergodicity. A left (left and right) $O_{0}(H)$-quasi-invariant probability measure $\mu$ is said to be left (left and right) $O_{0}(H)$-ergodic, if $\mu(A)=1$ or 0 for every subset $A \in \mathfrak{B}$ satisfying $\mu(T \cdot A \ominus A)=0$ for all $T \in O_{0}(H)(\mu(T \cdot A \cdot S \ominus A)=0$ for all $T, S \in O_{0}(H)$ ), respectively. In virtue of Lemma 2, it is equivalent that $\mu$ takes only the values 0 or 1 on $\mathfrak{U}_{\infty}\left(\mathfrak{B}_{\infty}\right)$, respectively.

Corollary. Two left (left and right) $O_{0}(H)$-ergodic measures are equivalent, if and only if they agree on $\mathfrak{A}_{\infty}\left(\mathfrak{B}_{\infty}\right)$, respectively.

Proposition 2. Let $\mu$ and $\nu$ be left $O_{0}(H)$-quasi-invariant probability measures on $\mathfrak{B}$, and put $\lambda(B)=\int_{g \in O(H)} \mu(B g) d \nu(g)$ for all $B \in \mathfrak{B}$. Then $\lambda$ is left and right $O_{0}(H)$-quasi-invariant. Moreover, if $\mu$ and $\nu$ are left $O_{0}(H)$-ergodic, then $\lambda$ is left and right $O_{0}(H)$-ergodic.

Proof. Let $S \in O_{0}(H)$. Then we have $\lambda(B)=0 \Leftrightarrow \mu(B g)=0$ for $\nu$ a. e. $g \Leftrightarrow \mu(B g)=0$ for $L_{S} \nu$-a. e. $g \Leftrightarrow \lambda(B \cdot S)=\int \mu(B g) d L_{S} \nu(g)=0$. This shows that $\lambda$ is right $O_{0}(H)$-quasi-invariant. Left $O_{0}(H)$-quasi-invariance of $\lambda$ is clear. Next, let $\mu$ and $\nu$ be left $O_{0}(H)$-ergodic, and let $A \in \mathfrak{B}_{\infty}$. As $A g \in \mathfrak{A}_{\infty}$ for all $g \in O(H)$, we have $\mu(A g)=1$ or 0 for all $g \in O(H)$. Put $E=\{g \in O(H) \mid \mu(A g)=1\}$. Then it follows from $E \in \mathscr{A}_{\infty}$ that we have $\nu(E)=1$ or 0 . Hence $\lambda(A)=1$, if $\nu(E)=1$ and $\lambda(A)=0$, if $\nu(E)=0$.

Q.E.D.

Now we shall consider an ergodic decomposition of $O_{0}(H)$-quasiinvariant measures. Let $\mu$ be a probability measure on $\mathfrak{B}$. As $(O(H)$, $d)$ is a Polish space, so for any sub- $\sigma$-field $\mathfrak{A}$ of $\mathfrak{B}$, there exists a family of conditional probability measures on $\mathfrak{B}$ relative to $\mathfrak{A}\{\mu(g$, $\mathfrak{A}, \cdot)\}_{\mathfrak{g} \in O(H)}$ which satisfy (1) for each fixed $B \in \mathfrak{B}, \mu(g, \mathfrak{A}, B)$ is an $\mathfrak{U}$-measurable function and (2) $\mu(A \cap B)=\int_{A} \mu(g, \mathfrak{A}, B) d \mu(g)$ for all $A \in \mathfrak{A}$ and for all $B \in \mathfrak{B}$. 
Lemma 4. Under the above notation, we take an arbitrary $B \in \mathfrak{B}$ and fix it. Then for all $n, \mu(g, \mathfrak{A}, T \cdot B \cdot S)$ is a jointly $\mathfrak{A} \times \mathfrak{B}(O(n))$ $\times \mathfrak{B}(O(n))$-measurable function of variables $(g, T, S) \in O(H) \times O(n)$ $\times O(n)$, where $\mathfrak{B}(O(n))$ is a usual Borel field on $O(n)$.

Proof. Let $f$ be a continuous bounded function on $O(H)$. Put $h(g, T, S)=\int_{O(H)} f\left(T^{-1} \cdot t \cdot S^{-1}\right) \mu(g, \mathfrak{A}, d t)$. Then (1) for a fixed $(T, S)$ $\in O(n) \times O(n), h(g, T, S)$ is $\mathfrak{U}$-measurable of $g$ and (2) for a fixed $g \in O(H) h(g, T, S)$ is continuous on $O(n) \times O(n)$. Hence $h(g, T, S)$ is jointly-measurable. Next, if $f$ is an indicator function of a closed set $B$, then we see that $h(g, T, S)$ is again measurable, taking a family of bounded continuous functions $\left\{f_{n}\right\}, f_{n} \downarrow f$. Now a family of Borel subsets satisfying the assertion of this Lemma is a monotone class, and contains an algebra generated by closed sets by the above arguments. Thus it coincides with $\mathfrak{B}$.

Q. E. D.

Let $\mu$ be a left $O_{0}(H)$-quasi-invariant probability measure on $\mathfrak{B}$. First we shall ask for conditional probability measures relative to $\mathfrak{A}_{n}$, using the normalized Haar measure $d T$ on $O(n)$ for each $n$. We put $\mu_{n}(B)=\int_{T \in O(n)} \mu(T \cdot B) d T$ for all $B \in \mathfrak{B}$. Then we have $\mu_{n} \simeq \mu, \mu_{n}(A)=$ $\mu(A)$ for all $A \in \mathfrak{Q}_{n}$ and $\mu_{n}$ is $O(n)$-invariant. It follows that for all $A \in \mathfrak{N}_{n}$ and for all $B \in \mathfrak{B}$,

$$
\begin{aligned}
\mu( & A \cap B)=\int_{A \cap B} \frac{d \mu}{d \mu_{n}}(g) d \mu_{n}(g) \\
& =\int_{A} \int_{T \in O(n)} \chi_{B}(T \cdot g) \frac{d \mu}{d \mu_{n}}(T \cdot g) d T d \mu_{n}(g) \\
& =\int_{A} \int_{T \in O(n)} \chi_{B}(T \cdot g) \frac{d \mu}{d \mu_{n}}(T \cdot g) d T d \mu(g) .
\end{aligned}
$$

Since

$$
\int_{T \in O(n)} \chi_{B}(T \cdot g) \frac{d \mu}{d \mu_{n}}(T \cdot g) d T \equiv \mu\left(g, \mathfrak{X}_{n}, B\right)
$$

is an $\mathfrak{A}_{n}$-measurable function of $g$ for each fixed $B \in \mathfrak{B}$, so we have $\mu\left(g, \mathfrak{A}_{n}, O(H)\right)=1$ for $\mu$-a. e. $g$ and $\left\{\mu\left(g, \mathfrak{A}_{n}, \cdot\right)\right\}_{g \in O(H)}$ is the family of conditional probability measures relative to $\mathfrak{U}_{n}$. Let $A \in \mathfrak{U}_{\infty}$ and $B \in \mathfrak{B}$. Then

$$
\mu_{n}(A \cap B)=\int_{T \in O(n)} \mu(A \cap T \cdot B) d T
$$




$$
\begin{aligned}
& =\int_{T \in O(n)} \int_{A} \mu\left(g, \mathfrak{U}_{\infty}, T \cdot B\right) d \mu(g) d T \\
& =\int_{A} d \mu_{n}(g) \int_{T \in O(n)} \mu\left(g, \mathfrak{A}_{\infty}, T \cdot B\right) d T .
\end{aligned}
$$

Therefore by Fubini's theorem and Lemma 4,

$$
\int_{T \in O(n)} \mu\left(g, \mathfrak{U}_{\infty}, T \cdot B\right) d T \equiv \mu_{n}\left(g, \mathfrak{U}_{\infty}, B\right)
$$

are conditional probability measures of $\mu_{n}$ relative to $\mathfrak{U}_{\infty}$. Since it holds $\mu_{n} \simeq \mu$, applying general discussions for conditional probability measures, it is assured that there exists an $\Omega_{n} \in \mathfrak{A}_{\infty}$ with $\mu\left(\Omega_{n}\right)=1$ such that

$$
\mu_{n}\left(g, \mathfrak{A}_{\infty}, \cdot\right) \equiv \mu_{n}^{g} \simeq \mu\left(g, \mathfrak{A}_{\infty}, \cdot\right) \equiv \mu^{g}
$$

and the Radon-Nikodim derivative $\frac{d \mu^{g}}{d \mu_{n}^{g}}$ can be taken as $\frac{d \mu}{d \mu_{n}}$ for all $g \in \Omega_{n} . \quad$ As $\mu_{n}^{g}$ is $O(n)$-invariant, we conclude that for all $g \in$ $\cap_{n=1}^{\infty} \Omega_{n} \equiv \Omega_{0}, \mu^{g}$ is left $O_{0}(H)$-quasi-invariant. Moreover, from $\left(\mu^{g}\right)_{n}=$ $\mu_{n}^{g}$ we have for all $g \in \Omega_{n}$,

$$
\begin{aligned}
& \mu^{g}\left(t, \mathfrak{A}_{n}, B\right)=\int_{T \in O(n)} \chi_{B}(T \cdot t) \frac{d \mu^{g}}{d\left(\mu^{g}\right)_{n}}(T \cdot t) d T \\
& \quad=\int_{T \in O(n)} \chi_{B}(T \cdot t) \frac{d \mu}{d \mu_{n}}(T \cdot t) d T \\
& \quad=\mu\left(t, \mathfrak{A}_{n}, B\right)
\end{aligned}
$$

for all $t \in O(H)$ and for all $B \in \mathfrak{B}$. Consequently, for all $g \in \Omega_{0}, \mu^{g}(t$, $\left.\mathfrak{A}_{n}, \cdot\right)=\mu\left(t, \mathfrak{A}_{n}, \cdot\right)$ holds for all $t \in O(H)$ and for all $n$. In virtue of inverse martingale theorem, for all $B \in \mathfrak{B}$,

$$
\begin{aligned}
0 & =\lim _{n} \int\left|\mu\left(t, \mathfrak{X}_{\infty}, B\right)-\mu\left(t, \mathfrak{A}_{n}, B\right)\right| d \mu(t) \\
& =\lim _{n} \int\left|\mu\left(t, \mathfrak{X}_{\infty}, B\right)-\mu\left(t, \mathfrak{N}_{n}, B\right)\right| d \mu^{g}(t) d \mu(g) .
\end{aligned}
$$

Taking a subsequence $\left\{n_{j}\right\}$ if necessary, there exists an $\Omega_{B}^{1} \in \mathfrak{A}_{\infty}$ with $\mu\left(\Omega_{B}^{1}\right)=1$ such that for all $g \in \Omega_{B}^{1}$,

$$
\lim _{j} \int\left|\mu\left(t, \mathfrak{A}_{\infty}, B\right)-\mu\left(t, \mathfrak{A}_{n_{j}}, B\right)\right| d \mu^{g}(t)=0 .
$$

Hence again using the inverse martingale theorem, we have for all $g \in \Omega_{B}^{1} \cap \Omega_{0}$,

$$
\int i \mu\left(t, \mathfrak{A}_{\infty}, B\right)-\mu^{g}\left(t, \mathfrak{A}_{\infty}, B\right) \mid d \mu^{g}(t)=0 .
$$


It follows that

$$
\begin{aligned}
& \int\left|\mu\left(g, \mathfrak{A}_{\infty}, B\right)-\mu^{g}\left(t, \mathfrak{A}_{\infty}, B\right)\right|^{2} d \mu^{g}(t) d \mu(g) \\
& \quad=2 \int \mu\left(g, \mathfrak{N}_{\infty}, B\right)^{2} d \mu(g)-2 \int \mu\left(g, \mathfrak{A}_{\infty}, B\right) \int \mu^{g}\left(t, \mathfrak{A}_{\infty}, B\right) d \mu^{g}(t) d \mu(g)=0 .
\end{aligned}
$$

Thus there exists an $\Omega_{B}^{2} \in \mathfrak{A}_{\infty}$ with $\mu\left(\Omega_{B}^{2}\right)=1$ such that

$$
\int\left|\mu\left(g, \mathfrak{A}_{\infty}, B\right)-\mu^{g}\left(t, \mathfrak{A}_{\infty}, B\right)\right| d \mu^{g}(t)=0
$$

for all $g \in \Omega_{B}^{2}$. Finally we shall put $\Omega=\bigcap_{B \in \mathscr{F}} \Omega_{B}^{2}$, where $\mathscr{F}$ is a countable algebra generated by a countable open base of $(O(H), d)$. Then for all $g \in \Omega$, the above formula holds for every $B \in \mathfrak{B}$, so for all $A \in \mathfrak{U}_{\infty}$ and $B \in \mathfrak{B}$,

$$
\mu^{g}(A \cap B)=\int_{A} \mu^{g}\left(t, \mathfrak{X}_{\infty}, B\right) d \mu^{g}(t)=\mu^{g}(B) \mu^{g}(A) .
$$

Especially, we have $\mu^{g}(A)=1$ or 0 for all $A \in \mathfrak{A}_{\infty}$ and it implies $\mu^{g}$ is left $O_{0}(H)$-ergodic for all $g \in \Omega_{0} \cap \Omega$. We shall conclude these arguments with the following theorem.

Theorem 2. Let $\mu$ be a left $O_{0}(H)$-quasi-invariant probability measure on $\mathfrak{B}$. Then the conditional probability measures $\mu\left(g, \mathfrak{A}_{\infty}, \cdot\right)$ relative to $\mathfrak{A}_{\infty}$ are left $O_{0}(H)$-ergodic for $\mu-a . e . g$.

From Theorem 2, we can derive a following theorem called canonical decomposition in a quite similar way with it in pp. 372-373 in [5].

Theorem 3. Let $\mu$ be a left $O_{0}(H)$-quasi-invariant probability measure. Then there exist a family of probability measures $\left\{\mu^{\tau}\right\}_{\tau \in \boldsymbol{R}^{1}}$ on $\mathfrak{B}$ and a map $p$ from $O(H)$ to $\boldsymbol{R}^{1}$ which satisfy

(a) $\mu^{\tau}$ is left $O_{0}(H)$-ergodic for all $\tau \in \boldsymbol{R}^{1}$,

(b) for each fixed $B \in \mathfrak{B}, \mu^{\tau}(B)$ is $\mathfrak{B}\left(\boldsymbol{R}^{1}\right)$-measurable,

(c) $p^{-1}\left(\mathfrak{B}\left(\boldsymbol{R}^{1}\right)\right) \subset \mathfrak{U}_{\infty}$,

(d) $\mu\left(B \cap p^{-1}(E)\right)=\int_{E} \mu^{\tau}(B) d p \mu(\tau)$ for all $B \in \mathfrak{B}$ and $E \in \mathfrak{B}\left(\boldsymbol{R}^{1}\right)$,

(e) there exists $E_{0} \in \mathfrak{B}\left(\boldsymbol{R}^{1}\right), \mu\left(p^{-1}\left(E_{0}\right)\right)=1$ such that $\mu^{\tau_{1}}$ and $\mu^{\tau_{2}}$ are mutually singular for all $\tau_{1}, \tau_{2} \in E_{0}\left(\tau_{1} \neq \tau_{2}\right)$.

Ergodic decomposition of left and right $O_{0}(H)$-quasi-invariant mea- 
sures is carried out in parallel with the left case, only changing the integrals $\int_{O(n)} \cdots d T$ into double integrals $\iint_{O(n) \times O(n)} \cdots d T d S$. And the statements of Theorem 3 remains valid, changing "left" and $\mathfrak{U}_{\infty}$ into "left and right" and $\mathfrak{B}_{\infty}$, respectively.

\section{References}

[1] Meyer, P. A., Probability and potentials, Waltham Mass. Blaisdell Publ. Co., 1966.

[2] Parthasarathy, K. R., Probability measures on metric spaces, Academic Press, 1967.

[3] Shale, D., Invariant integration over the infinite dimension orthogonal group and related spaces, Trans. Amer. Math. Soc., 124 (1966), 148-157.

[4] Shimomura, H., On the construction of invariant measure over the orthogonal group on the Hilbert space by the method of Cayley transformation, Publ. RIMS, Kyoto Univ., 10 (1975), 413-424.

[5] , Ergodic decomposition of quasi-invariant measures, ibid., 14 (1978), 359381.

[6] Yamasaki, Y., Invariant measure of the infinite dimensional rotation group, ibid., 8 (1972) 131-140.

[7] - Projective limit of Haar measures on $O(n)$, ibid., 8 (1972), 141-149.

[8] Measures on infinite-dimensional spaces, Kinokuniya, 1978, in Japanese. 
